\title{
Passive immunization in the combat against infectious diseases (COVID-19 included)
}

\author{
Joanna Lasocka ${ }^{1} \odot$, Artur Bielawski²® ${ }^{\circledR}$, Elżbieta Lachert ${ }^{1} \odot$ \\ ${ }^{1}$ Institute of Hematology and Transfusion Medicine in Warsaw \\ ${ }^{2}$ „BIOMED-LUBLIN” Factory of Serum and Vaccines, S.A.
}

\begin{abstract}
Summary
Passive immunization (passive immunity, passive immunotherapy) consists in the transfer of specific antibodies to an unprotected individual with the purpose of protecting from infection or treatment of the disease. Passive immunization is acquired either naturally (maternal antibodies cross the placenta to reach the fetus) or artificially when the patient is transfused with high-titer antibodies of human or animal origin or developed by genetic engineering (monoclonal antibodies, MAb). The beginnings of passive immunization date back to the end of the XIX century when Emil Adolf von Behring was the first to use antibodies of animal origin in the treatment of diphtheria and tetanus. In Poland, the Lublin Factory of Serum and Vaccines established in 1951 launched an industrial-scale production of immune sera against diphtheria, scarlet fever, measles, meningitis and poliomyelitis (Heine Medin disease).

Passive immunization is relied on for prevention of infection, for treatment of immunodeficiency disorders (including hypogammaglobulinemia), acute infections and poisoning. Immunity derived from passive immunization lasts for a few weeks to about four months.

Since December 2019, the ongoing SARS-CoV-2 pandemic has initiated numerous randomized trials focused on determining the effectiveness of convalescent plasma therapy for COVID-19 infected patients. In order to minimize the occurrence of adverse reactions following the administration of convalescent plasma, attempts have been made to produce hyperimmune anti-SARS-CoV-2 globulin (hIVIG). It is also worthwhile to mention that there are ongoing clinical trials on anti-SARS-CoV-2 immunoglobulin concentrate developed by Polish researchers under the supervision of Professor Tomasiewicz and in cooperation with the Institute of Hematology and Transfusion Medicine in Warsaw as well as BIOMED-LUBLIN (Project No. 2020/ABM/COVID19/0036).
\end{abstract}

Key words: passive immunization, convalescent plasma, SARS-CoV-2, COVID-19, anti-SARS-CoV-2 immunoglobulin

J. Transf. Med. 2021; 14: 50-57

Correspondence address: dr n. med. Joanna Lasocka, Institute of Hematology and Transfusion Medicine, Indiry Gandhi Street 14, 02-776 Warszawa, phone (22) 3496 386, e-mail: jlasocka@ihit.waw.pl

Translation: mgr Krystyna Dudziak

This article is available in open access under Creative Common Attribution-Non-Commercial-No Derivatives 4.0 International (CC BY-NC-ND 4.0) license, allowing to download articles and share them with others as long as they credit the authors and the publisher, but without permission to change them in any way or use them commercially. 


\section{Passive immunization}

Passive immunization, passive immunity or passive immunotherapy are all terms that refer to prophylactic or therapeutic transfer of specific antibodies to an unprotected individual.

Passive immunization is either:

- natural - when maternal antibodies cross the placenta to reach the fetus;

- artificially acquired - when high-titer antibodies (human or animal) specific to an infectious agent or toxin are given as a medication to induce immunity in a non-immune patient.

Passive immunization serves to reduce the risk of contracting an infectious disease or to relieve disease symptoms. Passive immunization is performed in patients who are unable to synthesize antibodies or were exposed to a disease to which they are not immune.

\section{Natural passive immunization}

A natural form of passive immunotherapy is cross-placental transfer of maternal IgG immunoglobulins to fetal blood. The crossing is mediated by Fc IgG receptors present on the surface of trophoblast cells. Breast milk provides IgA antibodies to the suckling infant which mainly affect mucous membranes of the digestive tract [1]. IgM antibodies already appear in the fetal period, while the production of IgG antibodies begins shortly after birth to reach about $60 \%$ of the titer detected in adults after 12 months. With time, maternal antibody titer gradually decreases to disappear completely when the newborn is about 9 months old. IgG antibody titer is at its lowest when the newborn is $2-3$ months old. Maternal antibodies effectively protect newborns and infants against most infectious diseases. The most striking example are cases of children with severe combined immunodeficiency (SCID) presenting clinical symptoms usually 6 months after birth i.e. when maternal antibodies are at the lowest $[2,3]$.

Another proof of the relation between maternal immunity and infant immunity is the similar prevalence of antibodies in infants below 1 month and that observed in 20-40 year old adults who represent the main age group of the mothers [4].

\section{Artificial passive immunization}

Short-term artificial passive immunity is acquired through administration of human or animal plasma antibodies, monoclonal antibodies (MAb), human immunoglobulin for intravenous (IVIG) or intramuscular (IMIG) use, specific im- munoglobulins from immunized donors or as result of convalescent plasma therapy (CPT).

Passive immunization provides protection against infection and it is used to treat immunodeficiency (hypogammaglobulinemia), acute infections and poisoning. Effects of passive immunization last from several weeks to approximately four months.

\section{History of passive immunization}

The first successful attempts at applying passive immunization in the treatment of diphtheria and tetanus (in animal studies) were published in 1890 in Deutsche Medizinische Wochenschrift, German Medical Journal [5]. The method was fairly quickly modified and adapted to clinical use. As early as the mid-1890s, diphtheria-specific antitoxin was successfully used in hospital setting to reduce mortality rate during diphtheria outbreaks. In 1901, Emil Adolf von Behring was awarded the first Nobel Prize in Medicine for applying serotherapy in the fight against diphtheria. The Nobel Committee emphasized the importance of von Behring's work by adding the following words to the official statement: "with the work (of von Behring) a new pathway in medicine has been opened, and physicians were given an important tool/weapon in their combat against disease and death".

In Germany alone immunotherapy (diphtheria specific agents) helped to save about 45000 patients annully. In the $1890 \mathrm{~s}$, the mortality rate for hospitalized diphtheria patients ranged from $47 \%$ to $60 \%$ and in the pre-antibiotic era the study outcome of Emil von Behring and his collaborator Shibasaburo Kitasato was the only hope for diphtheria patients.

Behring and Kitasato conducted similar experiments with Clostridium tetani. Rabbit blood immunized with Clostridium tetani gave mice complete protection against typically lethal doses of virulent bacteria and botulinum toxin. The study outcome gave rise to the production of specific anti-tetanus immunoglobulin [5].

In the first years of passive immunotherapy, due to the fact that it was impossible to obtain human antibodies on a large scale, attempts were made to obtain them from animals. Anti diphteria antibodies for example were obtained from immunized cows [6]. Although the technology of obtaining specific immunoglobulins has now been significantly modified, some specific immunoglobulins are still of animal origin, e.g. Antivenin, by MERC \& $\mathrm{Co}$ - an immunoglobulin of equine origin that neutralizes neurotoxin of the poisonous black widow spider (Latrodectus mactans), botulism 
antitoxin — bivalent ) types A and B (equine), Sanofi Pasteur used for prophylactic and therapeutic management of botulism.

The classic approach to antibody production consists in immunizing animals with a specific antigen and isolating the antibodies from the host's blood. Despite advanced engineering technology proteins of animal origin may induce numerous adverse reactions. Passive immunotherapy based on animal antibodies should therefore be applied only under strict medical supervision.

Passive immunization was used extensively for treatment of diseases such as scarlet fever and whooping cough from the 20 s to the 70 s of the $\mathrm{XX}$ century [7].

Medicinal products containing human immunoglobulin are used, among others, for treatment of primary immunodeficiency with impaired antibody production, diphtheria, tetanus, hepatitis $\mathrm{A}$ and $\mathrm{B}$ (HAV, HBV), rabies, measles, smallpox, primary immune thrombocytopenia, Kawasaki syndrome, lower respiratory tract infection and in prevention of feto-maternal incompatibility and hemolytic disease of the fetus and newborn.

Prophylactic administration of antibodies against hepatitis B has largely been replaced by vaccination; however, immunoprophylaxis of hepatitis $B$ infection is still indicated in cases of accidental exposure of unprotected individuals (incomplete or unknown immunization status) such as high risk hospitalized patients, sexual partners of individuals with acute hepatitis B infection or healthcare workers exposed to hepatitis B infected blood or other body fluids following percutaneous needle injury or cut. Immunoprophylaxis of hepatitis B infection is also applied to infants born to $\mathrm{HBsAg}$ positive mothers [8].

Human immunoglobulins reduce the symptoms of some systemic inflammatory and autoimmune diseases or induce remission of disease.

The method of plasma fractionation into proteins to obtain blood-derived medicinal products was developed by Cohn and his collaborators in the 1940's and is used even now in modified form [9]. Source material for fractionation is fresh frozen plasma (FFP) collected at 23 Polish Blood Transfusion Centers (CKiK). Plasma for fractionation is subjected to rigorous procedures such as screening for HBV, HCV and HIV infection markers, to reduce the risk of transmitting infection. Nevertheless the risk cannot be completely eliminated and therefore, since the 1980 s, both plasma and medicinal products from pooled plasma are subjected to pathogen inactivation.
Plasma used for manufacture of medicinal products which contain human immunoglobulins differs with regard to the titer of specific antibodies. The choice of plasma for a specific disease is determined by the optimal therapeutic effect to be achieved. One such medicinal product that has been taken into account is immunoglobulin for intravenous use (IVIG).

With advancement in technology and particularly in monoclonal antibody production techniques new methods were developed which largely reduced the demand for antibodies of animal origin.

An alternative approach to antibody production is the antibody Phage Display technology based on genetic engineering of bacteriophages and generating libraries comprising myriads of variants of recombinant proteins displayed on their surface. Phage libraries allow to isolate functional antibody fragments of virtually any specificity. The method was first described by Georg Smith in $1985[10,11]$.

Phage libraries are based on single chain fragment variables $(\mathrm{scFv})$ - the smallest functional antibody fragments responsible for interaction with the antigen.

Antibody libraries are generated by preparing template cDNA from B cells of non-immunized human donors and amplification of $\mathrm{V}_{\mathrm{H}}$ and $\mathrm{V}_{\mathrm{L}}$ antibody fragments or antibody construction based on random insertion of amino acid mutations in complementary determining regions (CDRs). A pool of various sequence- antibody variants is thus generated which are displayed on the surface of the phages. Screening of phage libraries allows to select (from phage pools) the specific phage that interacts with the selected antigen [12].

Antibody variants acquired through Phage Display technique may additionally be subjected to the affinity maturation process._This technique mimics the natural process of antibody production with increasing affinity for an antigen. A similar effect is attained in laboratory conditions/in vitro by developing second-generation libraries comprising variants of higher affinity to antigen [13].

The antibody fragments acquired as result of selection are screened by enzyme-linked immunosorbent assay (ELISA) and then sequenced to identify the most common variable regions used by the immune system to elicit antibody response. Antibody fragments produced by bacterial colonies are typically purified by affinity chromatography. Through analysis of selected antibodies it is possible to determine the precise constant kinetics of the antigen-antibody binding. 
In the same way $\mathrm{scFv}$ format antibodies are produced against haptens, proteins, carbohydrates, receptors, tumor antigens or viruses. They find application in therapy or in a variety of diagnostic kits.

Antibodies for therapeutic/clinical use may also be linked with cytostatic drugs (antibody-drug conjugates, ADCP) or radioisotopes for more effective delivery to target tissues. Phage Display technology is successfully used by many pharmaceutical companies in their scientific quest for biological drugs. Examples of commercially available human therapeutic antibodies obtained with Phage Display technique are: Humira (adalimumab) - an antibody directed against tumor necrosis factor $\alpha(\mathrm{TNF} \alpha)$ which - among others - is applied in the management of rheumatoid arthritis and Benlysta (belimumab) - human IgG1 directed against the B lymphocyte stimulator (BLyS), a soluble ligand of the TNF family cytokines, which are used in the treatment of systemic lupus erythematosus. Under clinical trials are other antibodies from phage libraries [14].

The validity and effectiveness of Phage Display technique for obtaining high-specificity human antibodies of therapeutic potential have been confirmed. Other technologies have also been developed based on chimeric monoclonal antibodies in which the mouse antibody is "humanized". The effect is achieved by replacing murine immunoglobulin genes with human immunoglobulin genes [15].

\section{Passive immunization in prevention of infectious diseases; Polish experience}

The significance of passive immunotherapy in the combat against infectious diseases was most striking after World War II when the situation in Poland became dramatic due to military operations, migration of people and troops. Immediate actions to prevent the spread of infectious diseases were required.

In 1944 a group of professors and scientists from university centers of Lviv, Warsaw and Poznań, founded the National Institute of Hygiene (PZH) with the seat in Lublin the main task of which was development, implementation and supervision of activities related to protection of the Polish population against the spread of infectious diseases.

In 1951 the Department for Production of Serum and Vaccines - one of the organizational units of $\mathrm{PZH}$ - was transformed into the Lublin Factory of Serum and Vaccines later known as "Biomed" Serum and Vaccine Production Plant PLC in Lublin ("BIOMED-LUBLIN" Wytwórnia Surowic i Szczepionek S.A) which is active until the pre- sent. It was the first biotech pharmaceutical plant in Poland. The immunopreparations developed and manufactured there were the crucial element of the national prophylactic strategy for years. The plant was also the first in Poland as well as in Central Europe to launch an industrial-scale production of immune sera against diphtheria, meningitis, measles and scarlet fever.

Anti-tetanic, anti-diphtheric, and anti-meningitis sera were prepared from blood of hyperimmunized horses. Measles were treated with human convalescent measles serum.

It would be difficult to overestimate the importance of the above-mentioned sera in the fight against tetanus, diphtheria, scarlet fever, measles or poliomyelitis (Heine Medin disease) in Poland. Currently, the production of immunoglobulins for treatment of the diseases is terminated as the latter are under control.

\section{Immunoglobulin (IVIG) for intravenous use and specific immunoglobulin for COVID-19 therapy}

IVIG in the United States was approved for clinical use in 1980 and found effective in prophylaxis of life-threatening infections in patients with primary and secondary immunodeficiencies. IVIG is used in the management of such chronic infections as parvovirus B19.

The current experience with IVIG in the treatment of SARS-CoV-2 infection is rather limited. The rationale for using IVIG in SARS-CoV-2 infection is primarily to modulate inflammation. IVIG has been found to reduce the inflammatory response in severe SARS-CoV-2 infections. Chinese publications report the use of IVIG in severe cases of SARS-CoV-2 infections where normalization of fever and improvement of respiratory symptoms occurred. The results however cannot be considered conclusive as the patients also received antiviral drugs and steroids which may have interfered with the impact of immunoglobulins [16]. It can therefore be concluded that there is currently no clear evidence to support the rationale for use of IVIG in the management of diseases such as SARS-CoV, SARS-CoV-2 and MERS.

On the other hand, specific immunoglobulin obtained from donor plasma with high antibody titer to certain infectious agents has been successfully used in the treatment of numerous infections, caused e.g. by cytomegalovirus (CMV) or influenza virus (H1N1). Specific immunoglobulins have the advantage of being obtained from the plasma of donors seropositive to a specific infectious agent 
with antibody titers high enough to neutralize the infectious agent [17].

Effectivity of specific immunoglobulins has also been confirmed in the management of infections caused by other coronaviruses (SARS severe acute respiratory syndrome and MERS Middle East Respiratory Syndrome) [18]. Publications confirmed accelerated improvement of clinical parameters and significantly lower mortality rate for patients treated with convalescent plasma during management of progressive COVID-19 infections [19]. In 2009, prospective analyses confirmed the effectiveness of plasma (titer $\geq 1$ : 160) from H1N1 - infected individuals transfused to patients with severe H1N1 infection [20]. One of the studies also demonstrated the advantage of specific immunoglobulins over IVIG; patients were observed to have lower viral load and prolonged survival time following administration of immunoglobulin obtained from plasma of severe H1N1 survivors as compared to therapy with IVIG [21].

\section{Passive immunization with convalescent plasma in the management of viral infections}

Plasma collected from individuals with a history of viral infections (convalescent plasma, CP) has been used in clinical setting since the end of the 19 th century infections. Successful attempts at CPT were made during the Spanish flu pandemic of 1919 [22-24].

Convalescent plasma was used, among others in the treatment of measles, mumps, chicken pox, cytomegalovirus, and parvovirus B19 infections. The World Health Organization (WHO) also recommended CPT during the Ebola epidemic [25]. In 2009, during the influenza A pandemic caused by the H1N1 subtype, a significant reduction in mortality rate was observed in patients treated with convalescent plasma as compared to control (20.0\% vs. $54.8 \%$; $\mathrm{p}=0.01$ ) [20]. Likewise, CP used in the treatment of MERS-CoV (Middle East Respiratory Syndrome Coronavirus) and SARS (severe acute respiratory syndrome) gave better therapeutic results as compared to control [26, 27].

In the literature, CPT is reported to be effective and well tolerated. Serious adverse reactions are infrequent. Convalescent plasma is particularly effective in the treatment of various viral infections as it reduces mortality rate, lowers viremia, accelerates recovery and shortens hospitalization.

Crucial for the therapeutic effect of convalescent plasma are the neutralizing antibodies of su- fficiently high titer. The Virus Neutralization Test (VNT) is the gold standard for determining the neutralizing antibody titer. The classic test consists in preparation of cell culture susceptible to infection with a specific virus. A series of dilutions of the test serum (or a solution containing antibodies) is then prepared and mixed with the suspension containing a certain amount of infectious virus particles. The mixture is then added to the cell culture. After the incubation period, the result is read from each well for the presence of viral infection; assessment of the dilution at which the viral infection is inhibited. For some viruses, a microscopic evaluation of the cytopathic effect may include morphological change in the infected cells or lysis and appearance of plaques in place of voids. The final result, expressed as the neutralizing antibody titer in the test sample, represents the last dilution of the serum effectively neutralizing the contaminated culture as compared to control [28].

Neutralization tests are highly specialized, labor-intensive and time-consuming tests that require access to laboratories of adequate biosafety level. Difficult to standardize, they are sensitive to cell maturity and type. The number of centers performing such tests is limited. Suitability of donor plasma for passive immunization is routinely determined by serological tests. For SARS-CoV-2, the tests based on the $\mathrm{S} 1$ or RBD spike protein antigens are best correlated with the level of neutralizing antibodies.

It is known for a fact that viremia typically peaks during the first week of infection, while the primary immune response develops at the end of the second week. Hence, applying plasma in the early stage of infection may bring better therapeutic effects [29].

The effectivity of passive immunization decreases as the infection progresses. Complete protection against symptoms of the disease is attained through application of immunoglobulin prior to exposure. Passive immunization is highly effective when administered immediately after exposure, before the symptoms develop. Immunoglobulin therapy is less effective when applied after the onset of disease symptoms. Little or no clinical benefit is observed with immunoglobulin therapy applied in the late stage of the disease (Fig. 1).

\section{Passive immunization vs. COVID-19}

At the end of December 2019 pneumonia cases were detected in the city of Wuhan (China, Hubei province) attributed to an unknown coronavirus, 


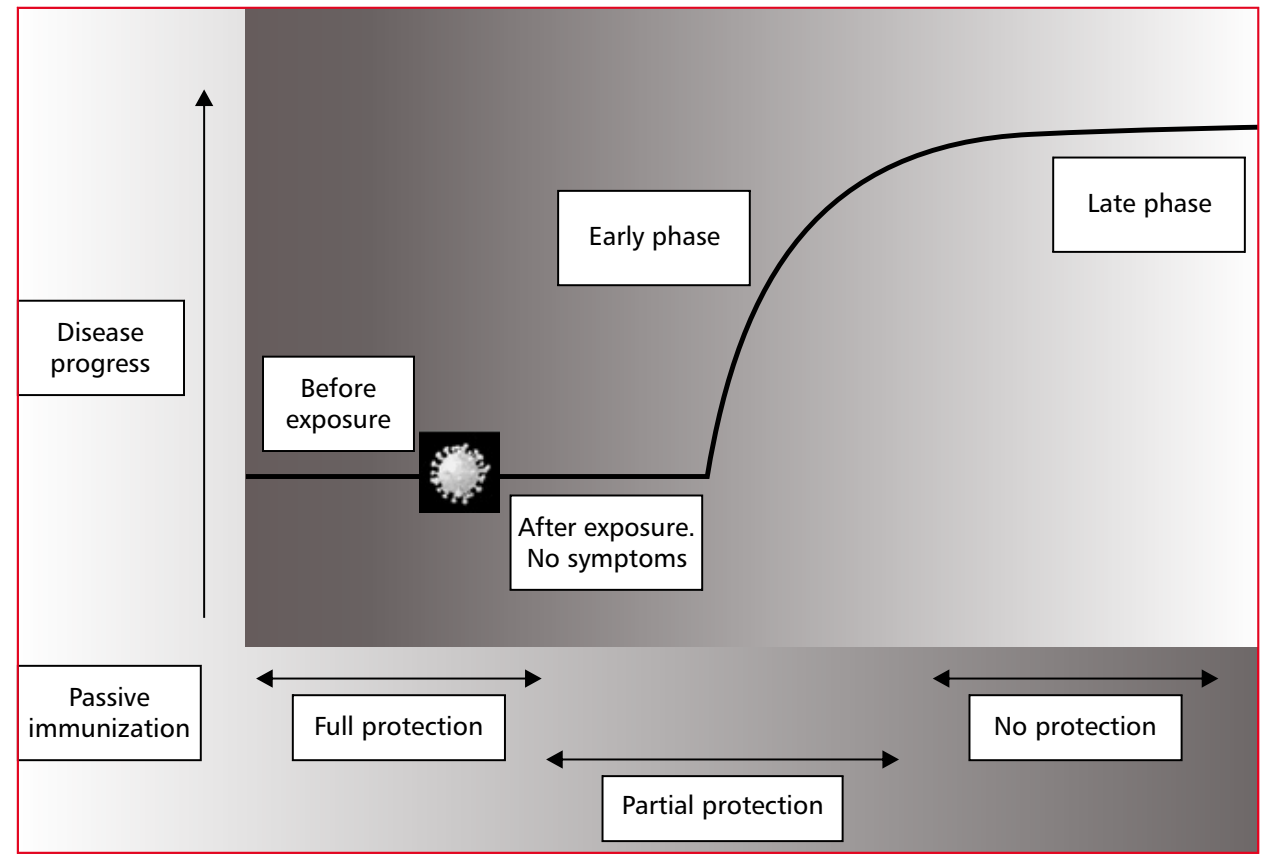

Figure 1. Effectivity of passive immunization with regard to disease phase

initially called 2019-nCoV. In February 2020, WHO approved the official name of the virus SARS-CoV-2 (Severe Acute Respiratory Syndrome Coronavirus 2) and of the disease - COVID-19 (Coronavirus Disease 2019). Coronaviruses are infectious agents classified into four types: $\alpha$ - and $\beta$-coronaviruses (infectious to mammals) and $\gamma$ - and $\delta$-coronaviruses (responsible for bird diseases). The SARS-CoV-2 virus (a $\beta$-coronavirus) is the seventh coronavirus known to infect humans and it owes the name to similarity to the human SARS virus $(82 \%)$ and to the SARS-like-CoVZXC21 in bats.

According to WHO estimates of April $18^{\text {nd }}$ 2021, 140322903 COVID-19 cases have been reported worldwide since the beginning of the pandemic and there were 3003794 fatal cases ascribed to SARS-CoV-2.

The COVID-19 pandemic caused by the spread of SARS-CoV-2 is both an unprecedented challenge for clinicians as well as a burden on the healthcare systems worldwide due to high infectivity and mortality rates. Given the rapid and catastrophic spread of COVID-19, the available treatment options had to be verified and investigated until a vaccine or other effective drug were developed. High hopes rested with plasma collected from COVID-19 survivors and immunoglobulin products.

Numerous randomized trials are underway to determine the efficacy of convalescent plasma therapy in COVID-19 infection [30].
According to recommendations of October $9^{\text {th }}$ 2020 of the American National Institutes of Health $(\mathrm{NIH})$ which conducts biomedical and health-related research, more than 70,000 patients in the US have already received CPT under the Mayo Clinic's Expanded Access Program (EAP) although data from well-controlled, randomized trials are still insufficient to demonstrate effectiveness and safety of CPT for COVID-19.

Retrospective, indirect clinical efficacy studies based on EAP data conducted by the Food and Drug Administration (FDA) and the Mayo Clinic demonstrated that patients who received high titer convalescent plasma presented better therapeutic effects than those who were given lower titer plasma. This was also confirmed by the results of Mayo Clinic with regard to mortality rate; patients mortality was lower $(22.3 \%)$ in the group that received high-titer convalescent plasma than in the group that received medium or low titer convalescent plasma; $27.4 \%$ and $29.6 \%$ [31] respectively. An exceptional response to therapy was observed when convalescent plasma was administered within 72 hours of COVID-19 diagnosis [32]. Adverse reactions for CPT are rare and comparable to administration of plasma for other indications.

Currently available scientific evidence confirms that the benefits of convalescent plasma in COVID-19 therapy outweigh the known and potential risks. 
In order to minimize side effects following CPT several centers worldwide have made attempts at manufacturing hyperimmune anti-SARS-CoV-2 globulin (hIVIG) [33]. The immunoglobulin concentrate allows to administer neutralizing antibodies in a smaller volume of plasma and that should help to minimize the number of adverse reactions related to transfusion. Various methods are used to produce immunoglobulin concentrate. Vandeberg et al relied on the chromatographic method which gave an approximately 10 fold higher concentration of specific anti-SARS-CoV-2 antibodies as compared to the concentration in pooled convalescent plasma while the neutralizing activity of the antibodies in the final product is about 3 fold higher than in the plasma pool [34]. A patient subjected to hIVIG therapy is administered higher titer antibodies (higher neutralizing activity) in a volume equivalent to that of convalescent plasma. The final product contains $100 \%$ IgG while unwanted proteins such as IgM, IgA, anti-A, anti-B, anti-D antibodies are almost completely eliminated. IgM antibodies responsible for the intravascular haemolytic activity of anti-A and anti-B antibodies were removed to prevent intravascular hemolysis [35]. So, unlike for plasma, selection of ABO-compatible donors is not required.

For IgA deficient patients who may have been treated with blood derived products and developed anti-IgA antibodies, removal of IgA guarantees better therapeutic outcome of hIVIG as compared to convalescent plasma.

The potential advantage of hIVIG over convalescent plasma lies in the diversity of antibodies obtained from convalescent plasma pool and the range of antiviral activity is therefore wider [36]. The diversity may be important given the emergence of new variants of the SARS-CoV-2 virus. Diverse antibodies provide a wider range of antiviral activity by targeting different virus epitopes through different cellular mechanisms [32].

hIVIG may therefore prove therapeutically more effective than fresh frozen plasma collected from COVID-19 survivors.

Clinical trials of products obtained under the supervision of Vanderberg are underway and may soon provide important information on the efficacy and safety of anti-SARS-CoV-2 hIVIG [37].

Polish clinical trials on anti-SARS-CoV-2 immunoglobulin concentrate conducted in cooperation with Biomed Lublin and the Institute of Hematology and Transfusion Medicine under the supervision of Professor Tomasiewicz (PROJECT No. 2020/ /ABM/COVID19/0036) are also worth mentioning.

\section{Future of passive immunization}

Significant advancement of monoclonal antibody technologies as well as the growing awareness of the role of antibodies in the combat against infectious diseases rationalizes further research into therapies based on passive immunization. Research into the development of antibody-based therapies is further strengthened by the emergence of antibiotic-resistant clinically significant bacteria (eg. Staphylococcus aureus or Salmonella typhi ). Antibody half-life in vitro is typically short therefore passive immunization provides only temporary protection and needs to be repeated. Innovative technologies to extend the half-life of monoclonal antibodies raise high hopes. One such example is the mutation of the Fc region of an anti-RSV monoclonal antibody to increase its binding to the $\mathrm{Fc}$ receptor. This induces changes in the pharmacokinetics of human serum antibodies which increase to 100 from the typical 19-34 day half-life while maintaining virus-specific neutralizing activity. While passive immunization may be sufficient for prophylactic or therapeutic treatment of acute infection or disease in remission, long-term immunity requires active immunization with "engineered" vaccine. It should be emphasized that effective methods of passive immunization may serve as basis for the development of novel and high quality vaccines.

\section{References}

1. Niewiesk S. Maternal antibodies: clinical significance, mechanism of interference with immune responses, and possible vaccination strategies. Front Immunol. 2014; 5: 446, doi: 10.3389/ fimmu.2014.00446, indexed in Pubmed: 25278941.

2. Fischer A. Severe combined immunodeficiencies (SCID). Clin Exp Immunol. 2000; 122(2): 143-149, doi: 10.1046/j.1365-2249.2000.01359.x, indexed in Pubmed: 11091267.

3. Lederman HM, Winkelstein JA. X-linked agammaglobulinemia: an analysis of 96 patients. Medicine (Baltimore). 1985; 64(3): 145-156, indexed in Pubmed: 2581110.

4. Slifka M, Amanna I. Passive Immunization. Plotkin's Vaccines. 2018: 84-95.e10, doi: 10.1016/b978-0-323-35761-6.00008-0.

5. Ueber das Zustandekommen der Diphtherie-Immunität und der Tetanus-Immunität bei Thieren. DMW - Deutsche Medizinische Wochenschrift. 2009; 16(49): 1113-1114, doi: 10.1055/s-0029-1207589.

6. Winau F, Winau R. Emil von Behring and serum therapy. Microbes Infect. 2002; 4(2): 185-188, doi: 10.1016/s1286-4579(01)01526-x, indexed in Pubmed: 11880051.

7. Marano G, Vaglio S, Pupella S, et al. Convalescent plasma: new evidence for an old therapeutic tool? Blood Transfus. 2016; 14(2): 152 -157, doi: 10.2450/2015.0131-15, indexed in Pubmed: 26674811.

8. Charakterystyka produktu leczniczego Gamma anty-HBs 200, „BIOMED-LUBLIN” Wytwórnia Surowic i Szczepionek Spółka Akcyjna. 
9. Cohn EJ, Strong LE. Preparation and properties of serum and plasma proteins; a system for the separation into fractions of the protein and lipoprotein components of biological tissues and fluids. J Am Chem Soc. 1946; 68: 459-475, doi: 10.1021/ ja01207a034, indexed in Pubmed: 21015743.

10. Smith GP. Filamentous fusion phage: novel expression vectors that display cloned antigens on the virion surface. Science. 1985; 228(4705): 1315-1317, doi: 10.1126/science.4001944, indexed in Pubmed: 4001944.

11. Alkan SS. Monoclonal antibodies: the story of a discovery that revolutionized science and medicine. Nat Rev Immunol. 2004; 4(2): 153-156, doi: 10.1038/nri1265, indexed in Pubmed: 15040588 .

12. Ahmad ZA, Yeap SK, Ali AM, et al. scFv antibody: principles and clinical application. Clin Dev Immunol. 2012; 2012: 980250, doi: 10.1155/2012/980250, indexed in Pubmed: 22474489.

13. Steinwand M, Droste P, Frenzel A, et al. The influence of antibody fragment format on phage display based affinity maturation of IgG. MAbs. 2014; 6(1): 204-218, doi: 10.4161/mabs.27227, indexed in Pubmed: 24262918.

14. Lu RM, Hwang YC, Liu IJ, et al. Development of therapeutic antibodies for the treatment of diseases. J Biomed Sci. 2020; 27(1): 1, doi: 10.1186/s12929-019-0592-z, indexed in Pubmed: 31894001.

15. Marasco WA, Sui J. The growth and potential of human antiviral monoclonal antibody therapeutics. Nat Biotechnol. 2007; 25(12): 1421-1434, doi: 10.1038/nbt1363, indexed in Pubmed: 18066039.

16. Cao W, Liu X, Bai T, et al. High-dose intravenous immunoglobulin as a therapeutic option for deteriorating patients with coronavirus disease 2019. Open Forum Infect Dis. 2020; 7(3): ofaa102, doi: 10.1093/ofid/ofaa102, indexed in Pubmed: 32258207.

17. Jawhara S. Could intravenous immunoglobulin collected from recovered coronavirus patients protect against COVID-19 and strengthen the immune system of new patients? Int J Mol Sci. 2020; 21(7), doi: 10.3390/ijms21072272, indexed in Pubmed: 32218340.

18. Who Mers-Cov Research Group. State of knowledge and data gaps of middle east respiratory syndrome coronavirus (MERS-CoV) in Humans. PLoS Curr. 2013; 5, doi: 10.1371/currents. outbreaks.0bf719e352e7478f8ad85fa30127ddb8, indexed in Pubmed: 24270606.

19. Shen C, Wang Z, Zhao F, et al. Treatment of 5 critically Ill patients with COVID-19 with convalescent plasma. JAMA. 2020; 323(16): 1582-1589, doi: 10.1001/jama.2020.4783, indexed in Pubmed: 32219428 .

20. Hung IFn, To KKw, Lee CK, et al. Convalescent plasma treatment reduced mortality in patients with severe pandemic influenza A (H1N1) 2009 virus infection. Clin Infect Dis. 2011; 52(4): 447-456, doi: 10.1093/cid/ciq106, indexed in Pubmed: 21248066.

21. Luke TC, Casadevall A, Watowich SJ, et al. Hark back: passive immunotherapy for influenza and other serious infections. Crit Care Med. 2010; 38(4 Suppl): e66-e73, doi: 10.1097/ CCM.0b013e3181d44c1e, indexed in Pubmed: 20154602.
22. Gould EW. Human serum in the treatment of influenza bronchopneumonia N Y Med J. 1919; 109: 666-667.

23. Holst J. Convalescent serum in the treatment of influenza Nor Mag Laegevidenskaben. 1919; 80: 31-561.

24. Huff-Hewitt W. Human serum in influenza. Br Med J. 1919; 1: 575.

25. Griensven Jv, Edwards T, Lamballerie Xde, et al. Evaluation of convalescent plasma for Ebola virus disease in Guinea. N Engl J Med. 2016; 374(1): 33-42, doi: 10.1056/nejmoa1511812, indexed in Pubmed: 26735992.

26. Who Mers-Cov Research Group. State of Knowledge and Data Gaps of Middle East Respiratory Syndrome Coronavirus (MERS$-\mathrm{CoV}$ ) in Humans. PLoS Curr. 2013; 5, doi: 10.1371/currents. outbreaks.0bf719e352e7478f8ad85fa30127ddb8, indexed in Pubmed: 24270606.

27. Wong VWS, Dai D, Wu AKL, et al. Treatment of severe acute respiratory syndrome with convalescent plasma. Hong Kong Med J. 2003; 9(3): 199-201, indexed in Pubmed: 12777656.

28. https://microbenotes.com/neutralization-test-introduction-and-types.

29. Cheng Y, Wong R, Soo YOY, et al. Use of convalescent plasma therapy in SARS patients in Hong Kong. Eur J Clin Microbiol Infect Dis. 2005; 24(1): 44-46, doi: 10.1007/s10096-004-1271-9, indexed in Pubmed: 15616839.

30. Piechotta V, Iannizzi C, Chai KLi, et al. Convalescent plasma or hyperimmune immunoglobulin for people with COVID-19: a rapid review. Cochrane Database Syst Rev. 2020; 5: CD013600, doi: 10.1002/14651858.CD013600, indexed in Pubmed: 32406927.

31. Joyner MJ, Carter RE, Senefeld JW, et al. Convalescent plasma antibody levels and the risk of death from Covid-19. N Engl J Med. 2021; 384(11): 1015-1027, doi: 10.1056/NEJMoa2031893, indexed in Pubmed: 33523609.

32. Food and Drug Administration. EUA 26382: emergency use authorization (EUA) request; 2020.

33. https://www.nih.gov/news-events/news-releases/nih-clinical-trial-testing-hyperimmune-intravenous-immunoglobulin-plus-remdesivir-treat-covid-19-begins.

34. Vandeberg P, Cruz M, Diez JM. Production of anti-SARS-CoV-2 hyperimmune globulin from convalescent plasma bioRxiv 2020 . 11; 18: 388991, doi: https://doi.org/10.1101/2020.11.18.388991.

35. Flegel WA. Pathogenesis and mechanisms of antibody-mediated hemolysis. Transfusion. 2015; 55 Suppl 2: S47-S58, doi: 10.1111/ trf.13147, indexed in Pubmed: 26174897.

36. Liu L, Wang P, Nair M, et al. Potent neutralizing antibodies against multiple epitopes on SARS-CoV-2 spike. Nature. 2020; 584(7821): 450-456, doi: 10.1038/s41586-020-2571-7, indexed in Pubmed: 32698192.

37. Inpatient treatment with anti-coronavirus immunoglobulin (ITAC) [monograph on the internet]. clinicaltrials.gov; 2020. Available from: https://clinicaltrials.gov/ct2/show/NCT04546581). 